\title{
Novel Phenyl and Purine Substituted Derivatives of Quinazolinones: Synthesis, Antioxidant and Antibacterial Features
}

\author{
NAGARAJA NAIK ${ }^{*}$ and JAVARAPPA RANGASWAMY \\ Department of Studies in Chemistry, University of Mysore, Manasagangotri, \\ Mysore -570006, Karnataka, India \\ drnaikchem@gmail.com
}

Received 2 August 2017 / Accepted 21 August 2017

\begin{abstract}
A one-pot three-component protocol for the preparation of novel phenyl and purine substituted derivatives of quinazolinones through the reaction of $7 \mathrm{H}$-purin-6-amine, cyclohexane1,3-diketone and aromatic aldehydes in acetic acid is reported. A broad range of quinazolinones were obtained in $55-82 \%$ overall yield. In this article, we describe the identification of the good oxidation inhibitors ( $\mathrm{IC}_{50}$ values of $13,16,22$ and $25 \mu \mathrm{M}$, respectively). Compounds IVf and IVg exhibited excellent in vitro radical inhibition activity against a DPPH radical, providing new opportunities for the series. In vitro antibacterial activity toward bacterial strain was also examined, compounds IVb, IVc and IVi exhibits stronger bacterial activity. Overall, the obtained results suggest that further optimization of activity of the series could provide a strong lead for a new antioxidant and antibacterial drug development program.
\end{abstract}

Keywords: Novel phenyl and purine substituted derivatives of quinazolinones, Antioxidant, Antibacterial activity

\section{Introduction}

The one-pot synthesis of a target molecule in the same reaction vessel is widely considered to be an efficient approach in synthetic organic chemistry. It is effective because several synthetic transformations and bond-forming steps can be carried out in a single pot, while circumventing several purification procedures at the same time. A one-pot procedure can thus minimize chemical waste, save time and simplify practical aspects. In fact, this approach has been used widely in synthetic organic chemistry for a long time ${ }^{1}$.

Heterocyclic chemistry comprises at least half of all organic chemistry research worldwide. In particular, heterocyclic structures form the basis of many pharmaceutical, agrochemical and veterinary products ${ }^{2}$. Quinazolines (Figure 1 compounds (a) and (b)) are classes of fused heterocycles that are of considerable interest because of the diverse range of their biological properties, for example, anticancer, diuretic, anti-inflammatory, anticonvulsant and antihypertensive activities ${ }^{3-6}$. Webber et al., has been reported 2-amino-6- 
methyl-5-(pyridin-4-ylsulfanyl)-3H-quinazolin-4-one (c) ${ }^{7}$ as a nonclassical inhibitor of human and $E$. coli TS with the inhibitory binding constants (K) of 15 and $49 \mu \mathrm{M}$, respectively. This compound further showed high cytotoxic activity against tumor cells in culture.<smiles>[R]c1nc2ccccc2c(=O)n1[R]</smiles>

(a)<smiles>[R]c1nc([R])c2ccccc2n1</smiles>

(b)<smiles>Cc1ccc2nc(N)[nH]c(=O)c2c1Sc1ccncc1</smiles>

(c)

Figure 1. General structure of bioactive quinazolines

On the basis of the promising initial data and also due to our interests in synthesis of biologically active heterocycles ${ }^{8-11}$, here a library of novel analogues was synthesized. Along with identifying the structural parameters that govern the antioxidant and antibacterial properties.

To the best of our knowledge, there was no report on the synthesis, antioxidant and antibacterial activity of phenyl and purine substituted derivatives of quinazolinones. To test our hypothesis, we designed and synthesized a series of phenyl and purine substituted derivatives of quinazolinones (Figure 2) and demonstrated their biological activity, some of new derivatives displayed excellent antioxidant and antibacterial activities. Herein, we report the detailed synthesis and biological evaluation of these compounds.

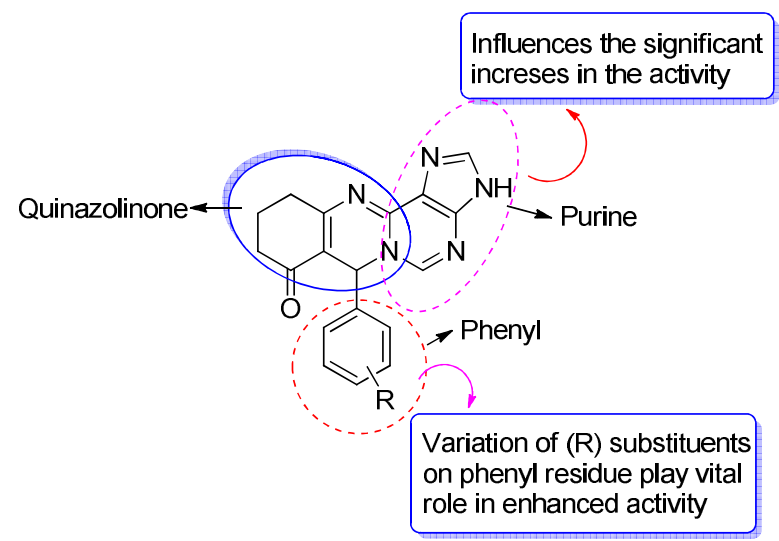

Figure 2. General structure of novel phenyl and purine substituted derivatives of quinazolinones (IVa-j)

\section{Experimental}

All reagents and solvents were purchased from Merck (Darmstadt, Germany) chemical AR grade and were used as provided. DPPH (2,2-diphenyl-1-picrylhydrazyl) was purchased from Sigma-Aldrich chemical Co. (St. Louis, MO, USA). TLC analysis was performed on alumina sheets precoated with silica gel $60 \mathrm{~F}-254$ and $\mathrm{SiO}_{2}, 200-400$ mesh (Merck) was used for column chromatography. ${ }^{1} \mathrm{H}$ NMR $(300 \mathrm{MHz}),{ }^{13} \mathrm{C}$ NMR $(100 \mathrm{MHz})$ were obtained AC Bruker spectrometer in the appropriate (DMSO) solvent. Melting points (Table 1) were obtained on a reichert thermopan melting point apparatus, equipped with a microscope and are uncorrected. Mass spectra were obtained on an Electron Impact mass spectrometer at $70 \mathrm{ev}$ ionizing beam and using a direct insertion probe. Micro analytical data were obtained by elemental-Vario EL-III. 
Table 1. Chemical structures, yield and melting point of novel phenyl and purine substituted derivatives of quinazolinones (IVa-j)

\begin{tabular}{|c|c|c|c|}
\hline Compounds & $\mathrm{R}$ & Yield, $\%$ & Melting point, ${ }^{\circ} \mathrm{C}$ \\
\hline IVa & & 70.35 & $133-135$ \\
\hline IVb & & 82.15 & $129-131$ \\
\hline IVc & & 67.30 & $250-252$ \\
\hline IVd & & 73.33 & $265-267$ \\
\hline IVe & & 55.00 & $179-181$ \\
\hline IVf & & 58.40 & 194-196 \\
\hline IVg & & 69.60 & $198-200$ \\
\hline IVh & & 77.20 & $213-215$ \\
\hline IVi & & 67.00 & $255-257$ \\
\hline $\mathbf{I V j}$ & & 62.50 & $217-219$ \\
\hline
\end{tabular}

Procedure for synthesis of novel phenyl and purine substituted derivatives of quinazolinones (IVa-j)

The stirred solution of $7 \mathrm{H}$-purin-6-amine $(2 \mathrm{mmol})$ and cyclohexane-1, 3-diketone ( $2 \mathrm{mmol})$ and aromatic aldehydes $(2 \mathrm{mmol})$ in $8 \mathrm{~mL}$ glacial acetic acid was refluxed for 4 hours in a round bottom flask fitted with reflux condenser. The progress of the reaction was monitored by using TLC hexane: ethylacetate (8:2) used as mobile phase. After completion of the reaction, the reaction mixture was poured in crushed ice to give buff colored precipitate which was filtered, dried and crystallized in ethanol to give the pure solid product.

7-Phenyl-7,9,10,11-tetrahydropurino[6,1-b]quinazolin-8(3H)-one (Iva)

Buff coloured solid; IR $(\mathrm{KBr}) \lambda_{\max }\left(\mathrm{cm}^{-1}\right): 1715(\mathrm{C}=\mathrm{O}), 3062.40(\mathrm{Ar}-\mathrm{H}), 3852.35(\mathrm{~N}-\mathrm{H}) ;{ }^{1} \mathrm{H}$ NMR $(300 \mathrm{MHz})\left(\mathrm{DMSO}-d_{6}\right) \delta(\mathrm{ppm}): 12.6(\mathrm{~s}, 1 \mathrm{H}, \mathrm{N}-\mathrm{H}), 8.52(\mathrm{~s}, 1 \mathrm{H}, \mathrm{CH}=\mathrm{N}), 7.62(\mathrm{~s}, 1 \mathrm{H}$, CH-N), 7.33-7.35 (d, 2H, $J=6 \mathrm{~Hz}, \mathrm{Ar}-\mathrm{H}), 7.27-7.31$ (dd, 1H, $J=6 \mathrm{~Hz}, \mathrm{Ar}-\mathrm{H}), 7.20-7.22$ (d, $2 \mathrm{H}, J=6 \mathrm{~Hz}, \mathrm{Ar}-\mathrm{H}), 5.18(\mathrm{~s}, 1 \mathrm{H}, \mathrm{Ar}-\mathrm{CH}-\mathrm{N}), 2.94-2.97\left(\mathrm{t}, 2 \mathrm{H}, \mathrm{J}=9 \mathrm{~Hz}\right.$, cyclohexanone $o-\mathrm{CH}_{2}$ ), 
1.93-1.96(t, 2H, $J=9 \mathrm{~Hz}$, cyclohexanone $\left.p-\mathrm{CH}_{2}\right), 1.67\left(\mathrm{~m}, 2 \mathrm{H}\right.$, cyclohexanone $\left.m-\mathrm{CH}_{2}\right),{ }^{13} \mathrm{C}$ NMR (DMSO- $\left.d_{6} 100 \mathrm{MHz}\right) \delta$ ppm: 20.5, 32.7, 36.0, 62.4, 117.8, 126.7, 126.9, 130.5, 143.3, $144.8,147.6,150.3,154.9,156.2,198.9$; Mass $(\mathrm{m} / z \%)$ : $\mathrm{M}^{+} 317.13$; Anal.calcd. for $\mathrm{C}_{18} \mathrm{H}_{15} \mathrm{~N}_{5} \mathrm{O}: \mathrm{C}, 68.15 ; \mathrm{H}, 4.70 ; \mathrm{N}, 22.06$; Found: $\mathrm{C}, 68.13 ; \mathrm{H}, 4.76 ; \mathrm{N}, 22.07 \%$.

7-(4-Nitrophenyl)-7,9,10,11-tetrahydropurino[6,1-b]quinazolin-8(3H)-one (IVb)

Yellow solid; IR $(\mathrm{KBr}) \lambda_{\max }\left(\mathrm{cm}^{-1}\right): 1715(\mathrm{C}=\mathrm{O}), 3124(\mathrm{Ar}-\mathrm{H}), 3856(\mathrm{~N}-\mathrm{H}), 1500(\mathrm{~N}=\mathrm{O}) ;{ }^{1} \mathrm{H}$ NMR (DMSO- $\left.d_{6} 300 \mathrm{MHz}\right) \delta \mathrm{ppm}: 12.6(\mathrm{~s}, 1 \mathrm{H}, \mathrm{N}-\mathrm{H}), 8.50(\mathrm{~s}, 1 \mathrm{H}, \mathrm{CH}=\mathrm{N}), 7.62(\mathrm{~s}, 1 \mathrm{H}, \mathrm{CH}-$ $\mathrm{N}), 8.12-8.14(\mathrm{~d}, 2 \mathrm{H}, J=6 \mathrm{~Hz}, \mathrm{Ar}-\mathrm{H}), 7.49-7.51(\mathrm{~d}, 2 \mathrm{H}, J=6 \mathrm{~Hz}, \mathrm{Ar}-\mathrm{H}), 5.18$ (s,1H, Ar-CH$\mathrm{N}), 2.94-2.98\left(\mathrm{t}, 2 \mathrm{H}, J=12 \mathrm{~Hz}\right.$, cyclohexanone $\left.o-C_{2}\right), 1.96-1.99(\mathrm{t}, 2 \mathrm{H}, J=9 \mathrm{~Hz}$, cyclohexanone $\left.p-\mathrm{CH}_{2}\right), 1.67\left(\mathrm{~m}, 2 \mathrm{H}\right.$, cyclohexanone $\left.m-\mathrm{CH}_{2}\right) ;{ }^{13} \mathrm{C}$ NMR (DMSO- $d_{6} 100$ MHz) $\delta$ ppm: 20.5, 32.7, 36.0, 62.4, 117.8, 125.3, 128.3, 130.5, 144.8, 145.9, 147.6, 149.4, 150.3, 154.9, 156.2, 198.9, Mass $(m / z \%)$ : $\mathrm{M}^{+} 362.11$; Anal.calcd. for $\mathrm{C}_{18} \mathrm{H}_{14} \mathrm{~N}_{6} \mathrm{O}_{3}: \mathrm{C}, 59.65$; H, 3.91; N, 23.20; Found: C, 59.67; H, 3.89; N, 23.19\%.

7-(4-Chlorophenyl)-7,9,10,11-tetrahydropurino[6,1-b]quinazolin-8(3H)-one (IVc)

Buff coloured solid; IR (KBr) $\lambda_{\max }\left(\mathrm{cm}^{-1}\right): 1715(\mathrm{C}=\mathrm{O}), 3132(\mathrm{Ar}-\mathrm{H}), 3858(\mathrm{~N}-\mathrm{H}), 814$ $(\mathrm{C}-\mathrm{Cl}) ;{ }^{1} \mathrm{H}$ NMR (DMSO- $\left.d_{6} 300 \mathrm{MHz}\right) \delta \mathrm{ppm}: 12.6$ (s, 1H, N-H), 8.58 (s, 1H, CH=N), 7.60 (s, 1H, CH-N), 7.37-7.39 (d, 2H, J=6 Hz, Ar-H), 7.34-7.37 (d, 2H, J=9 Hz, Ar-H), 5.18 $(\mathrm{s}, 1 \mathrm{H}, \mathrm{Ar}-\mathrm{CH}-\mathrm{N}), 2.94-2.97\left(\mathrm{t}, 2 \mathrm{H}, \mathrm{J}=9 \mathrm{~Hz}\right.$, cyclohexanone $\left.o-\mathrm{CH}_{2}\right), 1.96-2.00(\mathrm{t}, 2 \mathrm{H}, J=12$ $\mathrm{Hz}$, cyclohexanone $\left.p-\mathrm{CH}_{2}\right), 1.67\left(\mathrm{~m}, 2 \mathrm{H}\right.$, cyclohexanone $\left.m-\mathrm{CH}_{2}\right) ;{ }^{13} \mathrm{C}$ NMR (DMSO- $d_{6} 100$ MHz) $\delta$ ppm: 20.5, 32.7, 36.0, 6.4, 117.8, 126.1, 128.6, 130.5, 132.3, 141.4, 144.8, 147.6, 150.3, 154.9, 156.2, 198.9; Mass $(m / z \%): \mathrm{M}^{+} 351.09$; Anal.calcd. for $\mathrm{C}_{18} \mathrm{H}_{14} \mathrm{ClN}_{5} \mathrm{O}: \mathrm{C}$, 61.48; H, 4.02; N, 19.95; Found: C, 61.46; H, 4.01; N, $19.91 \%$.

7-(4-Hydroxyphenyl)-7,9,10,11-tetrahydropurino[6,1-b]quinazolin-8(3H)-one (IVd) Buff colored solid; IR $(\mathrm{KBr}) \lambda_{\max }\left(\mathrm{cm}^{-1}\right): 1715(\mathrm{C}=\mathrm{O}), 3132(\mathrm{Ar}-\mathrm{H}), 3858(\mathrm{~N}-\mathrm{H}), 3600$ $(\mathrm{O}-\mathrm{H}) ;{ }^{1} \mathrm{H}$ NMR (DMSO- $\left.d_{6} 300 \mathrm{MHz}\right) \delta$ ppm: 12.55 (s, $\left.1 \mathrm{H}, \mathrm{N}-\mathrm{H}\right), 8.55(\mathrm{~s}, 1 \mathrm{H}, \mathrm{CH}=\mathrm{N}), 7.60$ (s, 1H, CH-N), 7.32-7.35 (d, 2H, J=9 Hz, Ar-H), 7.24-7.26 (d, 2H, J=6 Hz, Ar-H), 5.18 $(\mathrm{s}, 1 \mathrm{H}, \mathrm{Ar}-\mathrm{CH}-\mathrm{N}), 2.94-2.98\left(\mathrm{t}, 2 \mathrm{H}, J=12 \mathrm{~Hz}\right.$ cyclohexanone $\left.o-\mathrm{CH}_{2}\right), 1.92-1.96(\mathrm{t}, 2 \mathrm{H}, J=9$ $\mathrm{Hz}$ cyclohexanone $\left.p-\mathrm{CH}_{2}\right), 1.67\left(\mathrm{~m}, 2 \mathrm{H}\right.$, cyclohexanone $\left.m-\mathrm{CH}_{2}\right) ;{ }^{13} \mathrm{C}$ NMR (DMSO- $d_{6} 100$ MHz) $\delta$ ppm: 20.5, 32.7, 36.0, 62.4, 115.7, 117.8, 126.1, 130.5, 135.9, 144.8, 147.6, 150.3, 154.9, 156.2, 156.5, 198.9; Mass (m/z\%): $\mathrm{M}^{+} 333.12$; Anal.calcd. for $\mathrm{C}_{18} \mathrm{H}_{15} \mathrm{~N}_{5} \mathrm{O}_{2}: \mathrm{C}, 64.88$; H, 4.53; N, 21.05; Found: C, 64.86; H, 4.54; N, 21.01; O, 9.60\%.

\section{7-(4-Methoxyphenyl)-7,9,10,11-tetrahydropurino[6,1-b]quinazolin-8(3H)-one (IVe)}

Brown solid; IR (KBr) $\lambda_{\max }\left(\mathrm{cm}^{-1}\right): 1715(\mathrm{C}=\mathrm{O}), 3132(\mathrm{Ar}-\mathrm{H}), 3858(\mathrm{~N}-\mathrm{H}), 1100(\mathrm{C}-\mathrm{O})$; ${ }^{1} \mathrm{H}$ NMR (DMSO- $\left.d_{6} 300 \mathrm{MHz}\right) \delta \mathrm{ppm}: 13.0(\mathrm{~s}, 1 \mathrm{H}, \mathrm{N}-\mathrm{H}), 8.26(\mathrm{~s}, 1 \mathrm{H}, \mathrm{CH}=\mathrm{N}), 7.60(\mathrm{~s}, 1 \mathrm{H}$, CH-N), 7.55-7.57 (d, 2H, J= $6 \mathrm{~Hz}, \mathrm{Ar}-\mathrm{H}), 6.85-6.87$ (d, 2H, J= $6 \mathrm{~Hz}, \mathrm{Ar}-\mathrm{H}), 5.18$ (s,1H, ArCH-N), $3.83\left(\mathrm{~s}, 3 \mathrm{H}, \mathrm{CH}_{3}\right), 2.94-2.98\left(\mathrm{t}, 2 \mathrm{H}, \mathrm{J}=9 \mathrm{~Hz}\right.$, cyclohexanone $\left.o-\mathrm{CH}_{2}\right), 1.93-1.96(\mathrm{t}, 2 \mathrm{H}$, $J=9 \mathrm{~Hz}$, cyclohexanone $\left.p-\mathrm{CH}_{2}\right), 1.67\left(\mathrm{~m}, 2 \mathrm{H}\right.$, cyclohexanone $\left.m-\mathrm{CH}_{2}\right) ;{ }^{13} \mathrm{C}$ NMR (DMSO$\left.d_{6} 100 \mathrm{MHz}\right) \delta$ ppm: 20.5, 32.7, 36.0, 55.8, 62.4, 114.1, 117.8, 125.7, 130.5, 135.6, 144.8, 147.6, 150.3, 154.9, 156.2, 158.6, 198.9; Mass $(\mathrm{m} / \mathrm{z} \%): \mathrm{M}^{+} 347.14$; Anal.calcd. for $\mathrm{C}_{19} \mathrm{H}_{17} \mathrm{~N}_{5} \mathrm{O}_{2}$ : C, 65.69; H, 4.93; N, 20.16; Found: C, 65.67; H, 4.92; N, 20.17\%.

7-(4-Hydroxy-3-methoxyphenyl)-7,9,10,11-tetrahydropurino[6,1-b]quinazolin$8(3 \mathrm{H})$-one $(\boldsymbol{I} \boldsymbol{V f})$

Off white solid; IR (KBr) $\lambda \max \left(\mathrm{cm}^{-1}\right): 1715(\mathrm{C}=\mathrm{O}), 3132(\mathrm{Ar}-\mathrm{H}), 3858(\mathrm{~N}-\mathrm{H}), 3600(\mathrm{O}-\mathrm{H}), 1100$ $(\mathrm{C}-\mathrm{H}) ;{ }^{1} \mathrm{H}$ NMR (DMSO- $\left.d_{6} 300 \mathrm{MHz}\right) \delta$ ppm: $12.62(\mathrm{~s}, 1 \mathrm{H}, \mathrm{N}-\mathrm{H}), 8.56(\mathrm{~s}, 1 \mathrm{H}, \mathrm{CH}=\mathrm{N}), 7.63$ 
(s, 1H, CH-N), 6.67-6.69 (d, 2H, $J=6 \mathrm{~Hz}, \mathrm{Ar}-\mathrm{H}), 6.60-6.62$ (d, 2H, $J=6 \mathrm{~Hz}, \mathrm{Ar}-\mathrm{H}), 5.35$ (s, $1 \mathrm{H}, \mathrm{O}-\mathrm{H}) 5.18(\mathrm{~s}, 1 \mathrm{H}, \mathrm{Ar}-\mathrm{CH}-\mathrm{N}), 3.83\left(\mathrm{~s}, 3 \mathrm{H}, \mathrm{CH}_{3}\right), 2.94-2.97(\mathrm{t}, 2 \mathrm{H}, J=9 \mathrm{~Hz}$, cyclohexanone $\left.o-\mathrm{CH}_{2}\right), 1.96-2.00\left(\mathrm{t}, 2 \mathrm{H}, \mathrm{J}=12 \mathrm{~Hz}\right.$, cyclohexanone $\left.p-\mathrm{CH}_{2}\right), 1.67(\mathrm{~m}, 2 \mathrm{H}$, cyclohexanone $m-\mathrm{CH}_{2}$ ); ${ }^{13} \mathrm{C}$ NMR (DMSO- $\left.d_{6} 100 \mathrm{MHz}\right) \delta$ ppm: $20.5,32.7,36.0,56.1,62.7$, $112.4,115.4,117.8,118.4,130.5,136.9,144.8,146.7,147.3,147.6,150.3,154.9,156.2$, 198.9; Mass (m/z\%): $\mathrm{M}^{+} 363.13$; Anal.calcd. for $\mathrm{C}_{19} \mathrm{H}_{17} \mathrm{~N}_{5} \mathrm{O}_{3}$ : C, 62.81; H, 4.74; N, 19.30; Found: C, $62.80 ; \mathrm{H}, 4.72 ; \mathrm{N}, 19.27 \%$.

7-(4-Hydroxy-3,5-dimethoxyphenyl)-7,9,10,11-tetrahydropurino[6,1-b]quinazolin$8(3 \mathrm{H})$-one (IVg)

Off white solid; IR $(\mathrm{KBr}) \lambda_{\max }\left(\mathrm{cm}^{-1}\right): 1715(\mathrm{C}=\mathrm{O}), 3132(\mathrm{Ar}-\mathrm{H}), 3858(\mathrm{~N}-\mathrm{H}), 3605(\mathrm{O}-\mathrm{H})$, $1100(\mathrm{C}-\mathrm{O}) ;{ }^{1} \mathrm{H}$ NMR (DMSO- $\left.d_{6} 300 \mathrm{MHz}\right) \delta \mathrm{ppm}: 12.57$ (s, 1H, N-H), 8.58 (s, 1H, CH=N), $7.62(\mathrm{~s}, 1 \mathrm{H}, \mathrm{CH}-\mathrm{N}), 6.67-6.69(\mathrm{~d}, 2 \mathrm{H}, J=6 \mathrm{~Hz}, \mathrm{Ar}-\mathrm{H}), 6.62-6.65(\mathrm{~d}, 2 \mathrm{H}, J=9 \mathrm{~Hz}, \mathrm{Ar}-\mathrm{H})$, $5.35(\mathrm{~s}, 1 \mathrm{H}, \mathrm{O}-\mathrm{H}) 5.18(\mathrm{~s}, 1 \mathrm{H}, \mathrm{Ar}-\mathrm{CH}-\mathrm{N}), 3.83\left(\mathrm{~s}, 3 \mathrm{H}, \mathrm{CH}_{3}\right), 2.90-2.94(\mathrm{t}, 2 \mathrm{H}, J=12 \mathrm{~Hz}$, cyclohexanone $\left.o-\mathrm{CH}_{2}\right), 1.88-1.92\left(\mathrm{t}, 2 \mathrm{H}, \mathrm{J}=12 \mathrm{~Hz}\right.$, cyclohexanone $\left.p-\mathrm{CH}_{2}\right), 1.65(\mathrm{~m}, 2 \mathrm{H}$, cyclohexanone $\left.m-\mathrm{CH}_{2}\right) ;{ }^{13} \mathrm{C}$ NMR (DMSO- $\left.d_{6} 100 \mathrm{MHz}\right) \delta$ ppm: $20.5,32.7,36.0,56.1,63.0$, $104.7,117.8,130.5,135.4,137.9,144.8,147.2,150.3,154.9,156.2,198.9 ;$ Mass $(\mathrm{m} / \mathrm{z} \%): \mathrm{M}^{+}$ 393.14; $\mathrm{C}_{20} \mathrm{H}_{19} \mathrm{~N}_{5} \mathrm{O}_{4}: \mathrm{C}, 65.71 ; \mathrm{H}, 4.92 ; \mathrm{N}, 20.19$; Found: C, 65.69; H, 4.93; N, 20.16\%.

7-(p-Tolyl)-7,9,10,11-tetrahydropurino[6,1-b]quinazolin-8(3H)-one (IVh)

Brown solid; IR $(\mathrm{KBr}) \lambda_{\max }\left(\mathrm{cm}^{-1}\right): 1715(\mathrm{C}=\mathrm{O}), 3132(\mathrm{Ar}-\mathrm{H}), 3858(\mathrm{~N}-\mathrm{H}), 1500(\mathrm{C}-\mathrm{H}) ;{ }^{1} \mathrm{H}$ NMR (DMSO- $\left.d_{6} 300 \mathrm{MHz}\right) \delta \mathrm{ppm}: 12.6(\mathrm{~s}, 1 \mathrm{H}, \mathrm{N}-\mathrm{H}), 8.58(\mathrm{~s}, 1 \mathrm{H}, \mathrm{CH}=\mathrm{N}), 7.59(\mathrm{~s}, 1 \mathrm{H}, \mathrm{CH}-$ N), 7.11-7.13 (d, $J=6 \mathrm{~Hz}, 2 \mathrm{H}, \mathrm{Ar}-\mathrm{H}), 7.07-7.09$ (d, 2H, $J=6 \mathrm{~Hz}, \mathrm{Ar}-\mathrm{H}), 5.35$ (s,1H,O-H), 5.18 (s,1H, Ar-CH-N), 2.90-2.94 (t, 2H, cyclohexanone $\left.\mathrm{J}=6 \mathrm{~Hz}, o-\mathrm{CH}_{2}\right), 2.34\left(\mathrm{~s}, 3 \mathrm{H}, \mathrm{CH}_{3}\right), 1.92-$ $1.96\left(\mathrm{t}, 2 \mathrm{H}, \mathrm{J}=12 \mathrm{~Hz}\right.$, cyclohexanone $\left.p-\mathrm{CH}_{2}\right), 1.67\left(\mathrm{~m}, 2 \mathrm{H}\right.$, cyclohexanone $\left.m-\mathrm{CH}_{2}\right) ;{ }^{13} \mathrm{C} \mathrm{NMR}$ (DMSO- $\left.d_{6} 100 \mathrm{MHz}\right) \delta$ ppm: 205, 21.3, 32.7, 36.0, 62.4, 117.8, 126.8, 128.8, 130.5, 136.4, $140.3,144.8,147.6,150.3,154.9,156.2,198.9$; Mass $(m / z \%): \mathrm{M}^{+} 331.14 ; \mathrm{C}_{19} \mathrm{H}_{17} \mathrm{~N}_{5} \mathrm{O}: \mathrm{C}$, 68.88; H, 5.19; N, 21.14; Found: C, 68.87; H, 5.17; N, 21.13; O, 4.83.

\section{7-(3-Chlorophenyl)-7,9,10,11-tetrahydropurino[6,1-b]quinazolin-8(3H)-one (IVi)}

Light yellow solid; IR (KBr) $\lambda_{\max }\left(\mathrm{cm}^{-1}\right): 1713(\mathrm{C}=\mathrm{O}), 3145(\mathrm{Ar}-\mathrm{H}), 3866(\mathrm{~N}-\mathrm{H}), 808$ $(\mathrm{C}-\mathrm{Cl}) ;{ }^{1} \mathrm{H}$ NMR (DMSO-d $\left.d_{6} 300 \mathrm{MHz}\right) \delta \mathrm{ppm}: 12.5$ (s, 1H, N-H), $8.58(\mathrm{~s}, 1 \mathrm{H}, \mathrm{CH}=\mathrm{N}), 7.62$ (s, 1H, CH-N), 7.35-7.37 (d, 2H, J=6 Hz, Ar-H), 7.30-7.33 (d, 2H, J=9 Hz, Ar-H), 5.21 $(\mathrm{s}, 1 \mathrm{H}, \mathrm{Ar}-\mathrm{CH}-\mathrm{N}), 2.90-2.94\left(\mathrm{t}, 2 \mathrm{H}, J=12 \mathrm{~Hz}\right.$, cyclohexanone $\left.o-\mathrm{CH}_{2}\right), 1.96-2.00(\mathrm{t}, 2 \mathrm{H}, J=12$ $\mathrm{Hz}$, cyclohexanone $\left.\mathrm{p}-\mathrm{CH}_{2}\right), 1.68\left(\mathrm{~m}, 2 \mathrm{H}\right.$, cyclohexanone $\left.m-\mathrm{CH}_{2}\right) ;{ }^{13} \mathrm{C}$ NMR (DMSO- $d_{6} 100$ MHz) $\delta$ ppm: 20.3, 33.5, 36.8, 60.4, 117.4, 126.9, 129.4, 131.0, 131.3, 141.9, 144.8, 147.8, 150.0, 155.3, 156.7, 199.1; Mass $(m / z \%): \mathrm{M}^{+} 351.09$; Anal.calcd. For $\mathrm{C}_{18} \mathrm{H}_{14} \mathrm{ClN}_{5} \mathrm{O}: \mathrm{C}$, 61.44; H, 4.04; N, 19.93; Found: C, 61.46; H, 4.01; N, $19.91 \%$.

\section{7-(2-Hydroxyphenyl)-7,9,10,11-tetrahydropurino[6,1-b]quinazolin-8(3H)-one (IVj)}

Buff colored solid; IR $(\mathrm{KBr}) \lambda_{\max }\left(\mathrm{cm}^{-1}\right): 1722(\mathrm{C}=\mathrm{O}), 3143(\mathrm{Ar}-\mathrm{H}), 3848(\mathrm{~N}-\mathrm{H}), 3615$ $(\mathrm{O}-\mathrm{H}) ;{ }^{1} \mathrm{H}$ NMR (DMSO-d $\left.d_{6} 300 \mathrm{MHz}\right) \delta \mathrm{ppm}: 12.4(\mathrm{~s}, 1 \mathrm{H}, \mathrm{N}-\mathrm{H}), 8.53(\mathrm{~s}, 1 \mathrm{H}, \mathrm{CH}=\mathrm{N}), 7.61$ (s, 1H, CH-N), 7.34-7.37 (d, 2H, $J=9 \mathrm{~Hz}, \mathrm{Ar}-\mathrm{H}), 7.23-7.27$ (d, 2H, $J=6 \mathrm{~Hz}, \mathrm{Ar}-\mathrm{H}), 5.18$ $(\mathrm{s}, 1 \mathrm{H}, \mathrm{Ar}-\mathrm{CH}-\mathrm{N}), 2.92-2.96\left(\mathrm{t}, 2 \mathrm{H}, J=12 \mathrm{~Hz}\right.$ cyclohexanone $\left.o-\mathrm{CH}_{2}\right), 1.94-1.98(\mathrm{t}, 2 \mathrm{H}, J=9$ $\mathrm{Hz}$ cyclohexanone $\left.p-\mathrm{CH}_{2}\right), 1.63\left(\mathrm{~m}, 2 \mathrm{H}\right.$, cyclohexanone $\left.m-\mathrm{CH}_{2}\right) ;{ }^{13} \mathrm{C}$ NMR (DMSO- $d_{6} 100$ MHz) $\delta$ ppm: 20.7, 32.3, 36.1, 62.2, 116.1, 118.3, 126.4, 130.5, 135.4, 145.5, 147.9, 151.0, 155.3, 156.7, 156.8, 198.7; Mass $(m / z \%)$ : $\mathrm{M}^{+} 333.12$; Anal.calcd. for $\mathrm{C}_{18} \mathrm{H}_{15} \mathrm{~N}_{5} \mathrm{O}_{2}: \mathrm{C}, 64.85$; H, 4.52; N, 21.08; Found: C, 64.86; H, 4.54; N, 21.01\%. 


\section{Biological studies}

\section{Antioxidant activity}

The newly synthesized compounds were screened for their radical scavenging activities using 2,2-diphenyl-1-picrylhydrazyl (DPPH) radical scavenging activity.

\section{DPPH radical scavenging assay}

The DPPH radical scavenging effect was carried out according to the method first employed by Blois $^{12}$. Compounds at different concentrations were prepared in distilled ethanol, $1 \mathrm{~mL}$ of each compound solutions having different concentrations $(10 \mu \mathrm{M}, 50 \mu \mathrm{M}, 100 \mu \mathrm{M}, 200 \mu \mathrm{M}$ and $500 \mu \mathrm{M})$ were taken in different test tubes, $4 \mathrm{~mL}$ of a $0.1 \mathrm{mM}$ ethanol solution of DPPH was added and shaken vigorously. The tubes were then incubated in the dark room at RT for $20 \mathrm{~min}$. A DPPH blank was prepared without compound and ethanol was used for the baseline correction. Changes (decrease) in the absorbance at $517 \mathrm{~nm}$ were measured using a UV-Visible spectrophotometer and the remaining DPPH was calculated. The percent decrease in the absorbance was recorded for each concentration and percent quenching of DPPH was calculated on the basis of the observed decreased in absorbance of the radical. The radical scavenging activity was expressed as the inhibition percentage and was calculated using the formula:

$$
\text { Radical scavenging activity }(\%)=\left[\left(\mathrm{A}_{\mathrm{o}}-\mathrm{A}_{1}\right) / \mathrm{A}_{\mathrm{o}} \times 100\right]
$$

Where $A_{o}$ is the absorbance of the control (blank, without compound) and $A_{1}$ is the absorbance of the compound. $\mathrm{IC}_{50}$ values were calculated by liner regression algorithm.

\section{Antibacterial activity}

The antibacterial activities of newly synthesized compounds were determined by well plate method in Mueller-Hinton Agar ${ }^{13}$. The antibacterial activity was carried out against $24 \mathrm{~h}$ old cultures of bacterial strains. In this work, E. coli, S. aureus, B. subtilis and $S$. typhimurium were used to investigate the activity. The test compounds were dissolved in dimethyl sulfoxide (DMSO) at concentration of $1000 \mathrm{mg} / \mathrm{mL} .20 \mathrm{~mL}$ of sterilized agar media was poured into each pre-sterilized Petri dish. Excess of suspension was decanted and plates were dried by placing in an incubator at $37^{\circ} \mathrm{C}$ for an hour. About $60 \mathrm{~mL}$ of $24 \mathrm{~h}$ old culture suspension were poured and neatly swabbed with the pre-sterilized cotton swabs. Six millimeter diameter well were then punched carefully using a sterile cork borer and $30 \mathrm{~mL}$ of test solutions of different concentrations were added into each labeled well. The plates were incubated for $24 \mathrm{~h}$ at $37^{\circ} \mathrm{C}$. The inhibition zone that appeared after $24 \mathrm{~h}$, around the well in each plate were measured as zone of inhibition in $\mathrm{mm}$. Experiments were triplicates and standard deviation was calculated.

\section{Results and Discussion}

The reaction involves $7 H$-purin-6-amine with cyclic $\beta$-diketone and aromatic aldehydes in the presence of glacial acetic acid. The reaction is proceed with Knoevenagal condensation between cyclic $\beta$-diketone and an aromatic aldehydes in the initial step to from an $\alpha, \beta$-unsaturated ketone, which undergoes Michael-type addition with the neucleophilic endocyclic nitrogen of $7 H$-purin-6-amine under reflux. The adduct is then cyclized intramolecularly with loss of water molecule to give novel phenyl and purine substituted derivatives of quinazolinones $(\mathbf{I V a} \mathbf{a}-\mathbf{j})(\text { Scheme } 1 \& 2)^{14}$. These reactions have taken place in one flask in a domino manner and the enone system generated in situ immediately undergoes Michael-type addition with $7 \mathrm{H}$-purin-6-amine and subsequent cyclization. The structures of the compounds were elucidated by IR, ${ }^{1} \mathrm{H}$ NMR, ${ }^{13} \mathrm{C}$ NMR, mass and elemental analysis. 
<smiles>Nc1ncnc2nc[nH]c12</smiles><smiles>O=C1CCCC(=O)C1</smiles>

(II)

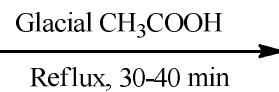

(I)

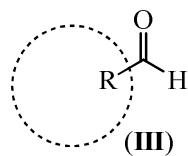

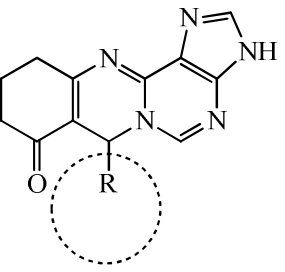

IV(a-j)

Scheme 1. Synthetic pathway for novel phenyl and purine substituted derivatives of quinazolinones (IVa-j)

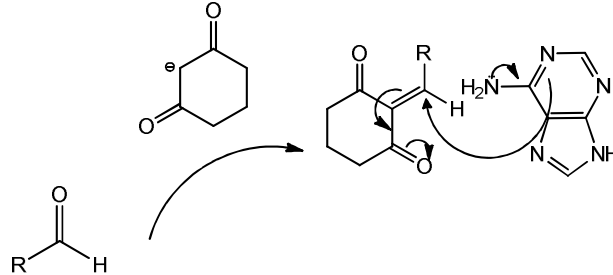

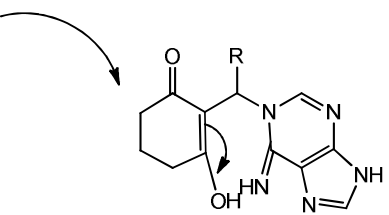<smiles>[R]C1=C2C(=O)CCCC2=NC2c3nc[nH]c3N=CN12</smiles><smiles>[R]C1[C@H]2C(=O)CCC[C@]2(C)C[C@]1(N)n1cnc2[nH]cnc21</smiles>

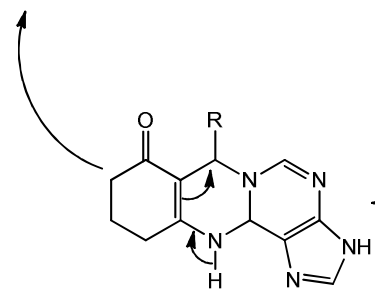<smiles>[13CH3]</smiles><smiles></smiles>

Scheme 2. Synthetic mechanism for novel phenyl and purine substituted derivatives of quinazolinones (IVa-j)

\section{Biological studies}

Antioxidant studies

Initially our basic compound I possess free N-H and $\mathrm{NH}_{2}$ electron donating groups showed considerable activity. Whereas, further reaction with substituted aldehydes and diketone gives the significant enhancement of antioxidant activity. From the Table 2, we can 
conclude that the antioxidant activity is depends on the position and type of functioning group presence on aldehydic phenyl ring. Among the synthesized compounds, IVd, IVf, IVg and IVj exhibited dominant activity. This may be due to the presence of electron donating methoxy, hydroxyl group at different position of phenyl ring. The compounds, IVe, IVh shown good activity but slightly less than the compounds IVd, IVf, IVg and IVj. This could be presence of single methoxy and methyl group on phenyl ring. Whereas, compounds IVb, IVc possess electron withdrawing like nitro and chloro, this might be the reason these compounds reveals less activity compare to other synthesized compounds. $\mathrm{IC}_{50}$ for all the synthesized compounds was also calculated and is depicted in Table 2. The decreasing orders of DPPH activity of newly synthesized analogues are as follows: IVg > IVf > IVd > ascorbic acid $>$ IVe $>$ IVh $>$ IVb $>$ IVe $>$ IVa.

Table 2. 50\% Inhibition of DPPH radical by compounds (IVa-j). Each value represents mean $\pm \mathrm{SD}(\mathrm{n}=3)$

\begin{tabular}{|c|c|}
\hline Compound & $\begin{array}{c}\mathrm{IC}_{50}[\mu \mathrm{M}] / \mathrm{mL} \\
\mathrm{DPPH}\end{array}$ \\
\hline IVa & $>500$ \\
\hline IVb & $200 \pm 0.21$ \\
\hline IVc & $300 \pm 0.05$ \\
\hline IVd & $22 \pm 0.13$ \\
\hline IVe & $101 \pm 0.17$ \\
\hline IVf & $14 \pm 0.09$ \\
\hline IVg & $11 \pm 0.23$ \\
\hline IVh & $170 \pm 0.35$ \\
\hline IVi & $400 \pm 0.03$ \\
\hline $\mathbf{I V j}$ & $25 \pm 0.57$ \\
\hline Ascorbic acid & $9 \pm 0.18$ \\
\hline
\end{tabular}

\section{Antibacterial activity}

The synthesized compounds (IVa-j) were also evaluated for their in vitro antibacterial activity against Staphylococcus aureus (Gram positive) and Escherichia coli (Gram negative) bacterial strains using well plate method in Mueller-Hinton Agar. As shown in our results, some analogues of this series were found to have near equal potency to the standard drug ciprofloxacin while some of them have least potency. Among the synthesized compounds IVb, IVc and IVi exhibited maximum bacterial inhibition power against tested microorganisms at a concentration of $1000 \mathrm{mg} / \mathrm{mL}$ similar to that of the standard ciprofloxacin. Whereas, compounds IVe and IVh displayed good antibacterial activity. The activity is considerably affected by substituents present at the para position of phenyl residue. The high potency of $\mathbf{I V b}, \mathbf{I V c}$ and IVi may be attributed to the presence of lipophilic or H-bond acceptor type group's placement of $\mathrm{Cl}$ and $\mathrm{NO}_{2}$ at 4-positions, respectively ${ }^{15}$. This might be the reason compounds IVb, IVc and IVi are highly active than other compounds. Rest of the compounds IVa, IVd, IVf-g, IVi-j bearing substituent such as $\mathrm{OH}, \mathrm{CH}_{3}$, and also having $\mathrm{OCH}_{3}$ groups at different position showed considerable or least activity with respect to standard drug against the tested strains.

It is clear from our results (Table 3) that the SAR of novel phenyl and purine substituted derivatives of quinazolinones (IVa-j) for bacterial inhibition effect strongly correlates with the substituent's at $C$-4 position of phenyl residue which favorable site for high inhibition effect. 
Table 3. Antibacterial activity of the compounds (IVa-j). Inhibitory zone (diameter) $\mathrm{mm}$ of the synthesized compounds against tested bacterial strains by well plate method

\begin{tabular}{ccc}
\hline \multirow{2}{*}{ Compound } & Gram positive & Gram negative \\
\cline { 2 - 3 } Control & Staphylococcus aureus & Escherichia coli \\
\hline $\mathbf{1}$ & $01 \pm 0.01$ & 0 \\
IVa & $01 \pm 0.02$ & $\mathrm{NA}$ \\
IVb & $19 \pm 0.10$ & $02 \pm 0.07$ \\
IVc & $17 \pm 0.01$ & $18 \pm 0.14$ \\
IVd & $03 \pm 0.21$ & $15 \pm 0.10$ \\
IVe & $10 \pm 0.01$ & $07 \pm 0.06$ \\
IVf & $19 \pm 0.21$ & $10 \pm 0.03$ \\
IVg & $02 \pm 0.17$ & $01 \pm 0.01$ \\
IVh & $11 \pm 0.23$ & $\mathrm{NA}$ \\
IVi & $15 \pm 0.34$ & $10 \pm 0.27$ \\
IVj & $02 \pm 0.10$ & $14 \pm 0.11$ \\
Ciprofloxacin & $22 \pm 0.32$ & $01 \pm 0.13$ \\
\hline
\end{tabular}

The concentration of test compounds was $1000 \mathrm{~g} / \mathrm{mL}$. Solvent used was DMSO. $N A=$ no active. The data represent mean value (SEM).

\section{Conclusion}

Synthesis, antioxidant and antibacterial properties of novel phenyl and purine substituted derivatives of quinazolinones (IVa-j) have been studied. Initially, a simple method used for the preparation of novel phenyl and purine substituted derivatives of quinazolinones by Knoevenagal condensation reaction with moderate to high yield. The antioxidant properties of the new analogues were evaluated by DPPH free radical scavenging activity. Among these compounds IVd, IVf, IVg reveals high radical scavenging activity near to the standard (ascorbic acid). Whereas, IVe, IVh shows good activity but less than the standard. Rest of compounds showed considerable to moderate radical scavenging activity. In vitro antibacterial activity toward gram positive and gram negative bacterial strain, compounds IVb, IVc and IVi exhibits stronger bacterial activity. Here substituents present on the phenyl residue is the important source for the significant increases in antioxidant and antibacterial property of synthesized analogues. This studies reveal that, substituent's electron donating and electron withdrawing groups on phenyl residue are crucial for the activity. On the basis of their activity, these derivatives were identified as viable leads for further studies.

\section{Acknowledgement}

The authors are grateful to the University Grants Commission, Government of India, for granting UGC SAP Phase III and UGC-Post Doctoral Fellowship-PDFSS.

\section{References}

1. Yujiro H, Chem Sci., 2016, 7, 866-880; DOI:10.1039/C5SC02913A

2. (a) David J C, Declan C, Timothy P O S and Patrick J G, Tetrahedron, 2005, 61, 10153-10202; DOI:10.1016/j.tet.2005.07.010; (b) Chan J H, Hong J S, Kuyper L F, Jones M L, Baccanari D P, Tansik R L, Boytos C M, Rudolph S K and Brown A D, J Heterocycl Chem., 1997, 34, 145-151; DOI:10.1002/jhet.5570340123

3. Gackenheimer S L, Schaus J M and Gehlert D R, J Pharmacol Exp Ther., 1996, 732, 113. 
4. Dempcy R O and Skibo E B, Biochemistry, 1991, 30(34), 8480-8487; DOI:10.1021/bi00098a028

5. Nordisk-Droge, Patent 18113, N. A. Ed.; Nordisk Drogeand Kemi-Kalieforretning AIS: Netherlands, 1965.

6. Webber S E, Bleckman T M, Attard J, Deal J G, Kathardekar V, Welsh K M, Webber S, Janson C A, Matthews D A, Smith W W, Freer S T, Jordan S R, Bacquet R J, Howland E F, Booth C L J, Ward R W, Hermann S M, White J, Morse C A, Hilliard J A and Bartlett C A, J Med Chem., 1993, 36, 733-746; DOI:10.1021/jm00058a010

7. Kumar H V, Kumar C K and Naik N, Med Chem Res., 2011, 20(1), 101-108; DOI:10.1007/s00044-009-9292-7

8. Rangaswamy J, Kumar H V, Harini S T and Naik N, J Heterocycl Chem., 2015, 52(3), 938-943; DOI:10.1002/jhet.1971

9. Harini S T, Kumar H V, Rangaswamy J and Naik N, Bioorg Med Chem Lett., 2012, 22(24), 7588-7592; DOI:10.1016/j.bmcl.2012.10.019

10. Rangaswamy J, Kumar H V, Harini S T and Naik N, J Heterocycl Chem., 2015, 52(5), 1349-1360; DOI:10.1002/jhet.2209

11. Rangaswamy J, Kumar H V, Harini S T and Naik N, Bioorg Med Chem Lett., 2012, 22(14), 4773-4777; DOI:10.1016/j.bmcl.2012.05.061

12. Blois M S, Nature., 1958, 26, 1199-1200; DOI:10.1038/1811199a0

13. Arthington-Skaggs B A, Motley M, Warnock D W and Morrison C J, J Clin Microbiol., 2000, 38(6), 2254-2260.

14. Maryam S, Mozhdeh S, Hamid R K and Ayoob B, Tetrahedron., 2008, 64(10), 23752378; DOI:10.1016/j.tet.2008.01.006

15. Rajesh H T, Atish H R, Girish D H and Ashish P K, Bioorg Med Chem Lett., 2011, 21, 4648; DOI:10.1016/j.bmcl.2011.03.062 